Journal of Mathematics and Statistics 5 (4): 283-286, 2009

ISSN 1549-3644

(C) 2009 Science Publications

\title{
An Extension of Yoshida, Imoto, Higuchi and Miyano Result
}

\author{
Onoghojobi B. \\ Department of Statistics, University of Ibadan, Nigeria
}

\begin{abstract}
Problem statement: Different approaches to deal with dynamic model when it is studentized are presented. Approach: In this respect, the recursive formula for calculating state space in the canonical form. Results: The asymptotic distribution of their test under the linear system for the gene network and a studentized-dynamic linear model with Markov denoising switching for estimating time-dependent gene network structure were presented. A resultant studentized version for gene network state space model was obtained, as an improvement on the original model. Conclusion/Recommendations: The dynamic model with Markov switching for estimating timedependent gene regulatory networks handled the problem of modeling change in an evolving time series. The studentized version incorporated the modeling change and test for heteroscedasticity.
\end{abstract}

Key words: Studentized-dynamic, Markov-denoising switching, studentized approach, recursive formula

\section{INTRODUCTION}

In this study we develop a new result for application of importance studentized method to state space with Markov switching for estimating gene regulatory network from time series microarray experiments. A draw back of dynamic linear model with Markov switching for estimating time seriesdependent gene network structure $\mathrm{s}(\hat{\theta})$ or (7) is that, it is crucially dependent on the assumption that $\varepsilon_{\mathrm{t}}$ is normally distributed. The general ideas of importance studentized method are well established in statistics and econometrics Cai, Hurvich and $\mathrm{Tsai}^{[2]}$, Koenker ${ }^{[10]}$, Yang and Tse ${ }^{[14]}$, Lyon and Tsai ${ }^{[11]}$, Deng and Perron ${ }^{[4]}$ and Silvia, Ferrari and Cribari-Neto ${ }^{[5]}$, following a robustification suggested by Bickel $^{[1]}$ we proposed a studentized version $\mathrm{s}(\hat{\omega})$.

Yoshida, Imoto and Higuchi ${ }^{[15]}$ proposed a simple method of estimating time-dependent gene network from time series microarray data by dynamic linear models with Markov switching and the state space version was later explored by Yamaguchi and Higuchi ${ }^{[13]}$ in which the state space model was represented by linear system for the gene network.

A state space model consists of a state equation and an observation equation. The state equation models the process of the state while the observation equation links the observations to the states. A standard linear state space model can be written as:

$$
\mathrm{X}_{\mathrm{t}}=\mathrm{F}_{\mathrm{t}} \mathrm{X}_{\mathrm{t}-1}+\eta_{\mathrm{t}}
$$

$$
\mathrm{Y}_{\mathrm{t}}=\mathrm{T}_{\mathrm{t}} \mathrm{X}_{\mathrm{t}}+\varepsilon_{\mathrm{t}}
$$

Where:

$\mathrm{X}_{\mathrm{t}}=$ The observation vector,

$\mathrm{F}_{\mathrm{t}}=$ The state transaction matrix and

$\mathrm{T}_{\mathrm{t}}=$ Observation matrix

It is assumed that $\eta_{t}$ and $\varepsilon_{t}$ are independent and identically distributed, with $\eta_{\mathrm{t}} \sim \mathrm{N}(0, \alpha)$ and $\varepsilon_{\mathrm{t}} \sim \mathrm{N}(0, \beta)$. The system matrices $\left(\mathrm{F}_{\mathrm{t}}, \mathrm{T}_{\mathrm{t}}\right)$ and the covariance matrices $(\alpha, \beta)$ may contain unknown parameter $\lambda$ and $\delta$ respectively.

The stationary linear problem ie when $\mathrm{F}_{\mathrm{t}}$ and $\mathrm{T}_{\mathrm{t}}$ are constant matrices solved by Wiener ${ }^{[12]}$ and Kailath ${ }^{[6]}$ and the non-stationary case was solved by Dacheng, Xu-Feng and Hulin ${ }^{[3]}$ Kalman $^{[7]}$, Kalman and Bucy ${ }^{[9]}$, Kalman $^{[8]}$. Yamaguchi and Higuchi ${ }^{[13]}$ and Yoshida, Imoto and Higuchi ${ }^{[15]}$ stated that if the true network structure underlying the data changes at certain point, the fitting of the usual dynamic linear model fail to estimate the structure of gene network, hence the efficient information from the data cannot be obtained. They therefore proposed a dynamic linear model with Markov switching time-dependent gene network structure for time series gene expression data, without taken into consideration the effect of studentizing the model as a test for heteroscedasticity. To solve this problem, we propose a studentized dynamic linear 
model with Markov switching for estimating time gene network from time series gene expression data $\mathrm{s}(\hat{\theta})_{\mathrm{s}}$. The new modification are necessary for conducting test for heteroscedasticity, estimation of parameters and their signal extraction of the state vector.

The study is organized as follows: we introduce the state model with Markov switching in details and derive a new score test to handle the state space model.

\section{MATERIALS AND METHODS}

Gene network state space model for linear system: The tools and logic for this reseasch are similar to the work of Yamaguchi and Higuchi ${ }^{[13]}$ and Yoshida, Imoto and Higuchi ${ }^{[15]}$, when dealing with estimating Time Series Dependent Gene Networks from Time Series Microarray Data. Also Yang and Tse ${ }^{[14]}$ for the tools and process of studentizing test. The state space model with Markov switching for estimating time dependent gene regulatory networks from time series microarray experiment is derived from a vector $r$ observed random variables $\mathrm{Y}_{\mathrm{t}}$ defined in (2) and $\mathrm{X}_{\mathrm{t}}$ in (1).

The state space model $\mathrm{Y}_{\mathrm{t}}$ in its canonical form implicitly represents a relationship $r$ genes across time point see Yoshida, Imoto, Higuchi ${ }^{[15]}$. The method is $Y_{t}$.

Let $\left(F_{t}, T_{t}, D_{t}\right)$ denote the model parameters depending on the time index, where:

$D_{t}=\operatorname{diag}\left\{d_{1} \ldots d_{r}\right\}$ of $\varepsilon_{t}$ such that:

$\mathrm{D}_{\mathrm{t}}^{-1 / 2} \mathrm{~T}_{\mathrm{t}}=\mathrm{L}_{\mathrm{t}} \Delta_{\mathrm{t}} \mathrm{U}_{\mathrm{t}}^{\prime}$

Where:

$\mathrm{L}_{\mathrm{t}}=$ The matrix of $\mathrm{p}$ orthogonal vector length $\mathrm{r}$

$\Delta_{\mathrm{t}}=$ The diagonal matrix that contain $\mathrm{p}$ singular values and $\mathrm{U}_{\mathrm{t}}^{\prime}$ is a $\mathrm{p} \times \mathrm{p}$ orthogonal matrix

The in verse of (3) is given as:

$\mathrm{T}_{\mathrm{t}}^{01}=\mathrm{U}_{\mathrm{t}} \Delta_{\mathrm{t}}^{-1} \mathrm{~L}_{\mathrm{t}}{ }_{\mathrm{t}}$

From Eq. 1 and 4:

$$
\begin{aligned}
& T_{t}^{01}\left(Y_{t}-\varepsilon_{t}\right)=\left(T_{t} X_{t}\right) T_{t}^{01} \\
& T_{t}^{01}\left(Y_{t}-\varepsilon_{t}\right)=T_{t} T_{t}^{01} X_{t} \\
& T_{t}^{01} D_{t}^{-1 / 2}\left(Y_{t}-\varepsilon_{t}\right)=X_{t}
\end{aligned}
$$

where, $\mathrm{T}_{\mathrm{t}} \mathrm{T}_{\mathrm{t}}^{01}=\mathrm{D}_{\mathrm{t}}^{1 / 2}$.

$\mathrm{T}_{\mathrm{t}}^{01} \mathrm{D}_{\mathrm{t}}^{-1 / 2}\left(\mathrm{Y}_{\mathrm{t}}-\varepsilon_{\mathrm{t}}\right)=\mathrm{F}_{\mathrm{t}} \mathrm{X}_{\mathrm{t}-1}+\eta_{\mathrm{t}}$

where, $X_{t-1}=T_{t-1}^{01} D_{t}^{-1 / 2}\left(Y_{t-1}-\varepsilon_{t-1}\right)+\eta_{t}$.

From Eq. 5 we have:

$\mathrm{T}_{\mathrm{t}}^{01} \mathrm{D}_{\mathrm{t}}^{-1 / 2}\left(\mathrm{Y}_{\mathrm{t}}-\varepsilon_{\mathrm{t}}\right)=\mathrm{F}_{\mathrm{t}} \mathrm{T}_{\mathrm{t}-1}^{01} \mathrm{D}_{\mathrm{t}-1}^{-1 / 2}\left(\mathrm{Y}_{\mathrm{t}-1}-\varepsilon_{\mathrm{t}-1}\right)+\eta_{\mathrm{t}}$

$F_{t}$ is known as the state transition matrix which captures the intensity of the interaction. By introduction of an interaction matrix $\mathrm{M}_{\mathrm{t}}$ such that $\mathrm{M}_{\mathrm{t}}=\mathrm{D}_{\mathrm{t}}^{-1 / 2} \mathrm{~T}_{\mathrm{t}} \mathrm{F}_{\mathrm{t}} \mathrm{T}_{\mathrm{t}}^{01}$ the state space model represent the linear system for the gene network as:

$\mathrm{D}_{\mathrm{t}}^{-1 / 2}\left(\mathrm{Y}_{\mathrm{t}}-\varepsilon_{\mathrm{t}}\right)=\mathrm{M}_{\mathrm{t}} \mathrm{D}_{\mathrm{t}-1}^{-1 / 2}\left(\mathrm{Y}_{\mathrm{t}-1}-\varepsilon_{\mathrm{t}-1}\right)+\mathrm{D}_{\mathrm{t}}^{-1 / 2} \mathrm{~T}_{\mathrm{t}} \eta_{\mathrm{t}}$

\section{RESULTS AND DISCUSSION}

Result of Studentized gene network state space model for linear system: The main analytical result of this study was obtained in (13) as shown below. Let $\mathrm{s}(\theta)$ denote (7) which can be rewritten as a score test:

$s(\hat{\theta})=T_{t}^{01}\left(Y_{t}-\varepsilon_{t}\right) T_{t}$

For details prove contact the author.

We begin by establishing a standard score test from (8). Hence, suppose that:

$\mathrm{s}(\hat{\theta})=\mathrm{T}_{\mathrm{t}}^{01} \mathrm{VT}_{\mathrm{t}}$

where, $V=\left(Y_{t}-\varepsilon_{t}\right)$.

Using the ideas of Cai et al. ${ }^{[2]}$, Koenter ${ }^{[10]}$, Yang and Tse $^{[14]}$, Deng and Perron ${ }^{[4]}$ and Silvia et al. ${ }^{[5]}$, let:

$\mathrm{s}(\hat{\theta})=\mathrm{T}_{\mathrm{t}}^{01} \mathrm{VT}_{\mathrm{t}}=\mathrm{s}(\hat{\omega}) \cdot 2 \sigma_{0}^{4}$

where, $s(\hat{\omega})$ is a standard score test Lyon and Tsai ${ }^{[2]}$, therefore:

$\mathrm{S}(\hat{\omega})=\frac{\mathrm{T}_{\mathrm{t}}^{01} \mathrm{~V} \mathrm{~T}_{\mathrm{t}}}{2 \sigma_{0}^{4}}$ 
Koenter ${ }^{[10]}$ proposed studentizing the statistic s $(\hat{\omega})$ by replacing $2 \sigma_{0}^{4}$ given in Eq. 11 with $\phi=\sum\left(\hat{e}_{\mathrm{i} 0}-\hat{\sigma}_{0}^{4}\right) / \mathrm{n}$. The resulting studentized score test can be obtained by substituting $\phi$ into Eq. 11 with regards to Eq. 10:

$s(\hat{\omega})_{s}=2 \hat{\sigma}_{0}^{4} s(\hat{\omega})$

Hence:

$s(\hat{\omega})_{s}=s(\hat{\theta}) / \phi$

Where:

$\mathrm{s}(\hat{\theta})=$ The original score test

$s(\hat{\omega})=$ The standard score test

$\mathrm{s}(\hat{\omega})_{\mathrm{s}}=$ The proposed studentized score test result.

\section{CONCLUSION}

The studentized test result in (13), has eliminated the draw back in the dynamic linear model with Markov Switching for estimating Time Series Dependent Gene structure in (7), the point raised by the studentized gene network might plausibly be considered common knowledge, except for the fact that proposing tests for heteroscedasticity based on Gaussian assumption for Markov switching are still being investigated.

The dynamic model with Markov switching for estimating time-dependent gene regulatory networks handled the problem of modeling change in an evolving time series. The studentized version incorporates the modeling change and test for heteroscedasticity. In this study, we established the studentized version of the dynamic system generated from the dynamic system itself using the technique based on Gibbs sampling. It is an improvement on the state space model with Markov switching for estimating time-dependent gene-regulatory networks from time series microarray experiments. We conclude that the proposed studentised gene network appear to be useful, flexible and accurate framework for dynamic model with Markov switching.

\section{REFERENCES}

1. Bickel, P.J., 1978. Using residuals robustly 1: Tests for heteroscedasticity, nonlinearity. Ann. Stat., 6: 266-291.

http://projecteuclid.org/DPubS? service=UI\&versio $\mathrm{n}=1.0 \&$ verb=Display\&handle=euclid.aos $/ 1176344$ 124

2. Cai, Z., C.M. Hurvich and C. Tsai, 1998. Score tests for heteroscedasticity in wavelet regression. Biometrika, 85: 229-234. DOI: 10.1093/biomet/85.1.229.

3. Dacheng, L., N. Xu-Feng and W. Hulin, 2006. Mixed-effects state space models for analysis of longitudinal dynamic systems. www.urmc.rochester.edu/smd/biostat/people/facult y/wu.html

4. Deng, A. and P. Perron, 2006. The limit distribution of CUSUM of square test under general mixing conditions. Unpublished manuscript Department of Economics, Boston University.

http://ideas.repec.org/p/bos/macppr/wp2006-004.html

5. Silvia, L., P. Ferraei and F. Cribarri-Neto, 2004 Beta regression for modeling rates and Proportions. J. Applied Stat., 31: 799-815, 2004. http://ideas.repec.org/a/taf/japsta/v31y2004i7p799815.html

6. Kailalh, T., 1981. Lectures on Wiener and Kalman Filtering. Springer Verlag Publishers, UK., ISBN: 038781664X, pp: 187.

7. Kalman, R.E., 1960. A new approach to linear filtering and prediction theory. J. Basic Eng. Trans. AMSE, Ser. D., 82: 32-45. http://www.cs.unc.edu/ welch/kalman/media/pdf/ Kalman1960.pdf

8. Kalman, R.E, 1963. Mathematical description of linear dynamical systems. SIAM J. Control, 1: 152-192. http://scitation.aip.org/getabs/servlet/GetabsServlet ?prog=normal\&id=SJCODC000001000002000152 000001\&idtype $=$ cvips\&gifs $=$ yes

9. Kalman, R.E. and R.S. Bucy, 1961. New results in linear filtering and prediction theory. Trans. AMSE Ser. D. J. Basic Eng., 83: 95-108. http://citeseer.comp.nus.edu.sg/context/57600/0

10. Koenker, R., 1981. A note on studentizing a test for heteroscedasitcity. J. Econometr., 17: 107-112. http://ideas.repec.org/a/eee/econom/v17y1981i1p1 07-112.html

11. Lyon, J. and C.L. Tsai, 1996. A comparison of testsfor heteroscedasticity. Statistican, 45: 337-349. http://cat.inist.fr/?aModele $=$ afficheN\&cpsidt $=3250$ 373 
12. Wiener, N., 1949. The Extrapolation, Interpolation and Smoothing of Stationary time Series with Engineering Applications Application: With Engineering Applications. The MIT Press, ISBN: 026223002X, pp: 163.

13. Yamaguchi, R. and T. Higuchi, 2006. State space Approach with maximum likehood principle to identify the system of generating time-course gene expression data yeast. Int. J. Data Mining Bioinform., $\quad 1$ : 77-87. DOI: 10.1504/IJDMB.2006.009922
14. Yang, Z. and Y. Tse, 2008. Generalized LM tests for functional form and heteroscedaticity. Econometr. J., 11: 349-376. DOI: 10.111/J.1368423X.2008.00242.X.

15. Yoshida, R., S. Imoto and T. Higuchi, 2005. Estimating time dependent gene networks from time series microarray data by dynamic linear models with Markov switching. Proceeding 4th Computation Systems Bioinformatics, Aug. 08-11, IEEE Computer Society Washington DC., USA., pp: 289-298. DOI: 10.1109/CSB.2005.32 